03

\title{
Гармоническая киноформная линза: дифракционная эффективность и хроматизм
}

\author{
(С) Г.И. Грейсух ${ }^{1}$, В.А. Данилов ${ }^{2}$, С.А. Степанов ${ }^{1}$, А.И. Антонов ${ }^{1}$, Б.А. Усиевич ${ }^{3}$ \\ ${ }^{1}$ Пензенский государственный университет архитектуры и строительства, \\ 440028 Пенза, Россия \\ ${ }^{2}$ Научно-технологический центр уникального приборостроения РАН, \\ 117342 Москва, Россия \\ ${ }^{3}$ Институт общей фризики им. А.М. Прохорова РАН, \\ 119991 Москва, Россия \\ e-mail: grey@pguas.ru
}

Поступила в редакцию 03.04.2018 г.

В окончательной редакции 24.04.2018 г.

В рамках скалярной и строгой теорий дифракции проведено исследование дифракционных линз с гармоническими пилообразными микроструктурами, имеющими глубину рельефа, существенно превышающую центральную длину волны рабочего спектрального диапазона. Показана возможность сохранения высокой дифракционной эффективности независимо от ширины рабочего спектрального диапазона и при допустимых углах падения излучения на линзу, модуль которых может достигать $45 \%$ и более. Показано также, что хроматизм таких линз управляем в весьма широких пределах и может оказаться меньшим, чем у самых легких кронов.

DOI: $10.21883 /$ OS.2018.08.46364.96-18

\section{Введение}

Гармонической сегодня называют киноформную линзу, пилообразная микроструктура которой работает в высоких порядках дифракции $(k \gg 1)$ и имеет глубину рельефа, существенно превышающую расчетную длину волны $[1,2]$. Апертура гармонической киноформной линзы разбита на так называемые гармонические зоны Френеля. Под этим термином понимается кольцевая зона апертуры, расстояния от краев которой до точки наблюдения (в данном случае фокальной точки $F$ ) различаются на величину, равную произведению целочис-

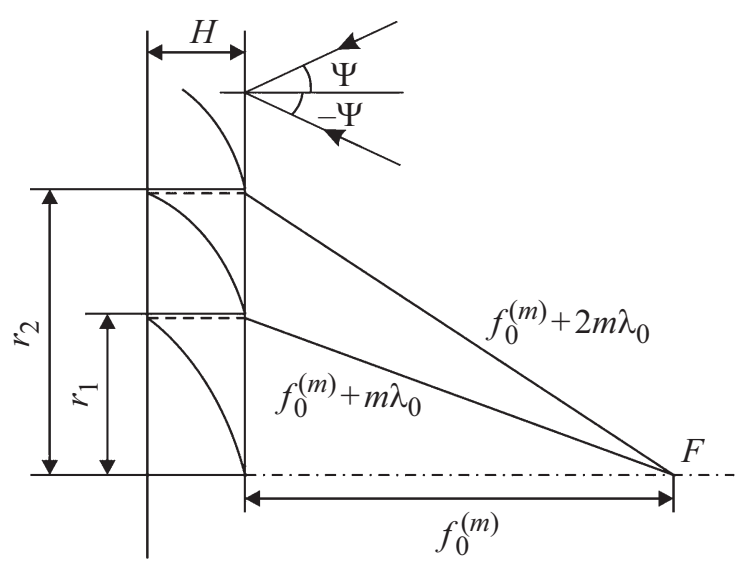

Рис. 1. Три приосевые зоны микроструктуры гармонической киноформной линзы. В верхней части рисунка показано, как соотносится знак угла падения излучения на линзу с пилообразным профилем микроструктуры при расчетах в рамках строгой теории дифракции. ленного порядка гармоничности $m$ на расчетную длину волны $\lambda_{0}$, как это показано на рис. 1.

Идеальная фокусировка нормально падающей на гармоническую киноформную линзу плоской монохроматической волны и при этом концентрация всей падающей на эту линзу энергии в единственном фокусе (единичная дифракционная эффективность (ДЭ)) достигаются при условии, что в пределах каждой гармонической зоны Френеля обеспечивается таутохронность, т.е. лучи, идущие от падающего волнового фронта до точки наблюдения, имеют одинаковую оптическую длину и, в частности, равны оптические длины лучей, проходящих через края каждой зоны. Если линза работает в $k$-м порядке дифракции, но $k=m$, то для $i$-й гармонической зоны Френеля $(i=1,2,3 \ldots)$ равенство оптических длин имеет вид

$$
H n_{\lambda_{0}}+f_{0}^{(m)}+(i-1) m \lambda_{0}=H+f_{0}^{(m)}+i m \lambda_{0},
$$

где $H$ - глубина $i$-й зоны, $n_{\lambda_{0}}$ и $f_{0}^{(m)}-$ показатель преломления материала подложки киноформной линзы и фокусное расстояние линзы в $m$-м дифракционном порядке на расчетной длине волны $\lambda_{0}$. Равенство оптических длин остальных лучей внутри каждой зоны обеспечивается за счет так называемого согласованного или коррелированного профиля пилообразного рельефа, впервые предложенного в работе [3].

Из уравнения (1) следует, что выполнение условия равенства оптических длин не зависит от фокусного расстояния, которое определяет радиусы гармонических зон. Действительно, из рис. 1 легко видеть, что у $i$-й зоны расстояние от центра микроструктуры линзы до 
внешнего края зоны

$$
r_{i}=\sqrt{2 i f_{0}^{(m)}+\left(i m \lambda_{0}\right)^{2}} .
$$

Вновь обращаясь к уравнению (1) и решая его относительно $H$, получим

$$
H=m \lambda_{0} /\left(n_{\lambda_{0}}-1\right)
$$

откуда, в частности, следует, что поскольку $H$ от $i$ не зависит, то глубины рельефа всех зон киноформной линзы должны быть одинаковыми.

Таким образом, гармоническая киноформная линза, преобразующая плоскую и нормально падающую на нее волну с длиной $\lambda_{0}$ в сферическую - это дифракционный оптический элемент с пилообразной рельефно-фазовой микроструктурой, характеризуемый, в частности, одновременным выполнением условий (2) и (3).

Пусть рабочая длина волны не совпадает с расчетной $\lambda \neq \lambda_{0}$. Требуя выполнения условия таутохронности для этой длины волны, найдем номер дифракционного порядка $k$ и фокусное расстояние $f_{\lambda}^{(k)}$, для которых условие таутохронности по-прежнему будет выполняться. Это условие для указанной длины волны и номера дифракционного порядка принимает вид

$$
H n_{\lambda}+f_{\lambda}^{(k)}+(i-k) k \lambda=H+f_{\lambda}^{(k)}+i k \lambda .
$$

И вновь видно, что выполнение требуемого условия не зависит от фокусного расстояния, и, следовательно, можно принять $f_{\lambda}^{(k)}=f_{\lambda}^{(m)}=f_{0}^{(m)}$.

Решив уравнение (4) с учетом формулы (3) относительно $\lambda$, получим уравнение для длины волны $\lambda_{k}$, на которой ДЭ в $k$-м дифракционном порядке будет равна единице:

$$
\lambda_{k}=\lambda_{0} \frac{m}{k} \frac{n_{\lambda}-1}{n_{\lambda_{0}}-1} .
$$

В случае традиционной киноформной линзы, т.е. при $m=1$, рабочий дифракционный порядок $k$ может принимать значения $1,2,3$ и т.д., а $\lambda_{k} \leq \lambda_{0}$. При этом наибольший интервал между двумя идеально фокусируемыми длинами волн $\Delta \lambda=\lambda_{1}-\lambda_{2} \cong 0.5 \lambda_{0}$. В результате, если расчетная длина волны $\lambda_{0}$ принадлежит видимому спектральному диапазону $\left(0.4 \leq \lambda_{0} \leq 0.7 \mu \mathrm{m}\right)$, то все $\lambda_{k}$, начиная с $k \geq 2$, оказываются вне этого диапазона. Принципиально иная ситуация в случае гармонической киноформной линзы с $m \gg 1$. Она допускает все варианты: $k=m, k>m$ и $k<m$. То есть, у гармонической киноформной линзы идеально фокусируемые длины волн $\lambda_{k}$ составляют линейчатый спектр с длинами волн как короче, так и длиннее $\lambda_{0}$ и к тому же с существенно меньшими и управляемыми, как будет показано ниже, интервалами между спектральными линиями. Очевидно, что этот характерный для гармонической киноформной линзы линейчатый спектр и определил ее название [1].

Зависимость показателя преломления оптического материала от длины волны, описываемая той или иной
Таблица 1. Основные параметры гармонических киноформных линз, выполненных из кроноподобной и флинтоподобной оптических пластмасс

\begin{tabular}{c|c|c|c|c|c}
\hline \multirow{2}{*}{$m$} & \multirow{2}{*}{$k$} & \multicolumn{2}{|c|}{ PMMA } & \multicolumn{2}{c}{ PC } \\
\cline { 3 - 6 } & & $\begin{array}{c}\lambda_{k}, \\
\mu \mathrm{m}\end{array}$ & $\begin{array}{c}\Delta \lambda=\lambda_{k}-\lambda_{k+1}, \\
\mu \mathrm{m}\end{array}$ & $\begin{array}{c}\lambda_{k}, \\
\mu \mathrm{m}\end{array}$ & $\begin{array}{c}\Delta \lambda=\lambda_{k}-\lambda_{k+1}, \\
\mu \mathrm{m}\end{array}$ \\
\hline \multirow{2}{*}{13} & 18 & 0.4074 & 0.0192 & 0.4163 & 0.0173 \\
\cline { 3 - 6 } & 10 & 0.7066 & 0.0615 & 0.6700 & 0.0592 \\
\hline \multirow{2}{*}{25} & 35 & 0.4034 & 0.0100 & 0.4125 & 0.0092 \\
\cline { 2 - 6 } & 19 & 0.7149 & 0.0344 & 0.7079 & 0.0332 \\
\hline \multirow{2}{*}{50} & 69 & 0.4086 & 0.0052 & 0.4174 & 0.0062 \\
\cline { 2 - 6 } & 38 & 0.7149 & 0.0176 & 0.7080 & 0.0170 \\
\hline \multirow{2}{*}{100} & 138 & 0.4086 & 0.0026 & 0.4174 & 0.0024 \\
\cline { 2 - 6 } & 76 & 0.7059 & 0.0087 & 0.6993 & 0.0084
\end{tabular}

дисперсионной формулой, в любом случае нелинейна, и решать уравнение (5) целесообразно итерационно. В табл. 1 сведены результаты решения этого уравнения, полученные при $\lambda_{0}=0.55 \mu \mathrm{m}$ для гармонических киноформных линз, выполненных из кроноподобного полиметилметакрилата (РММА) и флинтоподобного поликарбоната (РC) [4] и рассчитанных на работу в видимом спектральном диапазоне $0.4 \leq \lambda \leq 0.7 \mu \mathrm{m}$. Как видно из этой таблицы, при одном и том же порядке гармоничности интервал между двумя идеально фокусируемыми длинами волн $\Delta \lambda$ растет от коротковолнового края рабочего спектрального диапазона к длинноволновому, при этом крайние значения этой величины уменьшаются с ростом $m$.

\section{Дифракционная эффективность гармонической киноформной линзы}

Оценим ДЭ гармонической киноформной линзы на произвольной длине волны в рамках скалярной теории дифракции. Для этого воспользуемся известной формулой, приведенной в работе [5]:

$$
\eta=\left[\frac{\sin (\pi(k+\chi))}{\pi(k+\chi)}\right]^{2},
$$

где

$$
\chi=(H / \lambda)\left(\cos \theta-\sqrt{n_{\lambda}^{2}-\sin ^{2} \theta}\right),
$$

a $\theta-$ угол падения излучения из воздуха на элемент со стороны пилообразного рельефа. Учитывая формулу (3), получим

$$
\chi=\frac{m \lambda_{0}}{\lambda\left(n_{\lambda_{0}}-1\right)}\left(\cos \theta-\sqrt{n_{\lambda}^{2}-\sin ^{2} \theta}\right) .
$$


Подставив выражение (7) в (6), ДЭ запишем в виде

$$
\eta=\left(\frac{\sin \pi\left(k+\frac{m \lambda 0}{\lambda} \frac{\cos \theta-\sqrt{n_{\lambda}^{2}-\sin ^{2} \theta}}{\left(n_{\lambda_{0}}-1\right)}\right)}{\pi\left(k+\frac{m \lambda_{0}}{\lambda} \frac{\cos \theta-\sqrt{n_{\lambda}^{2}-\sin ^{2} \theta}}{\left(n_{\lambda_{0}}-1\right)}\right)}\right) .
$$

Зависимость ДЭ от длины волны при $\theta=0$ представлена на рис. 2.

Здесь следует обратить внимание на то, что независимо от каждого из параметров, входящих в выражение (8), кривые $\eta(\lambda)$ пересекаются на одной и той же высоте $\approx 0.4$. Если при этом учесть, что в формировании полезного изображения на любой длине волны $\lambda_{k+1} \leq \lambda \leq \lambda_{k}$ будет участвовать излучение, одновременно дифрагированное в оба указанных порядка, то график результирующей ДЭ будет иметь вид, представленный кривой 4 на рис. 2 . То есть, на каждой из длин волн, лежащих в промежутке между $\lambda_{k+1}$ и $\lambda_{k}$, ДЭ не опустится ниже 0.8 , а на побочные порядки, ответственные за падение контраста в изображении, гало и т.д., будет приходиться не более $20 \%$ падающей на гармоническую линзу энергии. Причем ДЭ не опустится ниже 0.8 независимо от полной ширины рабочего спектрального диапазона. Здесь уместно заметить, что у обычной киноформной линзы $(m=1)$ при нормальном падении излучения и равенстве ДЭ на краях видимого спектрального диапазона $(0.4 \leq \lambda \leq 0.7 \mu \mathrm{m})$ эта ДЭ на краях не может быть выше 0.75 . Следовательно, с точки зрения эффективности, рассчитанной в рамках скалярной теории дифракции, гармоническая линза даже в видимом диапазоне имеет определенное преимущество по сравнению с обычной киноформной.

Обратившись к выражениям (2) и (5), нетрудно видеть, что линейчатый спектр единичной ДЭ не совпадает со спектром, которому соответствует фиксированное фокусное расстояние $f=f_{0}^{(m)}$. Действительно, условие $f=f_{0}^{(m)}$ выполняется для длин волн $\lambda=m \lambda_{0} / k$, не зависящих от показателя преломления материала линзы, в то время как $\eta=1$ обеспечивается на длинах волн, в которые показатель преломления входит. О степени несовпадения позволяет судить табл. 2, в которой представлены длины волн, полученные при $\lambda_{0}=0.55 \mu \mathrm{m}$ и $m=50$ для гармонических киноформных линз, выполненных из РММА и РС. Данное несовпадение линейчатых спектров будет пусть и незначительно, но отрицательно влиять на качество изображения.

Далее покажем в рамках скалярной теории дифракции (СТД), какие изменения с ДЭ гармонической киноформной линзы происходят при изменении угла падения излучения на линзу. Из формулы (8) следует, что с ростом $\theta$ изменяются значения $k$, при которых в заданном интервале длин волн $\eta=1$. То есть в отличие от обычного у гармонического киноформа ДЭ, вычисляемая в рамках СТД, с ростом угла падения излучения на элемент не падает, а остается практически неизменной. Изменяются лишь номера дифракционных порядков, т. е.

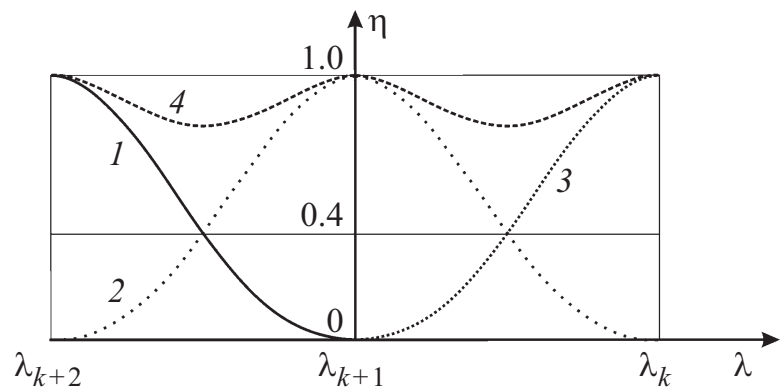

Рис. 2. Зависимости ДЭ от длины волны в дифракционных порядках $k(1), k+1(2)$ и $k+2(3) ; 4-$ кривая суммарной ДЭ двух соседних дифракционных порядков.

Таблица 2. Длины волн, обеспечивающие выполнение соответствующих критериев в выбранных дифракционных порядках

\begin{tabular}{l|c|c|c}
\hline \multirow{2}{*}{ Критерий } & \multirow{2}{*}{$k$} & \multicolumn{2}{|c}{$\lambda, \mu \mathrm{m}$} \\
\cline { 3 - 4 } & & PMMA & PC \\
\hline \multirow{3}{*}{$\eta=1$} & 50 & \multicolumn{2}{|c}{0.55} \\
\cline { 2 - 4 } & 51 & 0.5399 & 0.5403 \\
\cline { 2 - 4 } & 60 & 0.4639 & 0.4688 \\
\hline \multirow{3}{*}{$f=f_{0}^{(m)}$} & 50 & \multicolumn{2}{|c}{0.55} \\
\cline { 2 - 4 } & 51 & \multicolumn{2}{|c}{0.5392} \\
\cline { 2 - 4 } & 60 & \multicolumn{2}{|c}{0.4583} \\
\hline
\end{tabular}

если $\eta=1$ при нулевом угле падения излучения $(\theta=0)$ наблюдалось на какой-то длине волны $\lambda$ в $k^{\prime}$ порядке, то при угле $\theta>0 \eta=1$ (на примерно той же длине волны) передвинется на порядок $k^{\prime \prime}>k^{\prime}$. Однако следует учесть, что реальная ДЭ, которую позволяет достоверно прогнозировать лишь строгая теория дифракции, существенно зависит от отношения периода микроструктуры к глубине рельефа (например, [6]), и рост глубины с увеличением порядка гармоничности может привести к ощутимому падению ДЭ, особенно при больших углах падения излучения на микроструктуру.

Для исследования зависимости ДЭ пилообразной гармонической микроструктуры от длины волны и угла падения излучения на микроструктуру в рамках строгой теории дифракции, основанной на решении системы уравнений Максвелла, использовался строгий метод связанных волн [rigorous coupled-wave analysis (RCWA)] [7]. $\mathrm{B}$ соответствии с ним пилообразный рельеф дифракционной микроструктуры заменялся ступенчатым, и каждая ступень ограничивала тонкий слой, образующий бинарную диэлектрическую решетку, к которой применялось разложение в ряд Фурье. С использованием компьютерной программы, представленной в [8], исследовалась пилообразная гармоническая микроструктура, выполненная в оптической пластмассе РММА. В каче- 
Таблица 3. Номера дифракционных порядков и длины волн, на которые приходятся максимумы ДЭ, полученные в рамках СТД и RCWA-методом

\begin{tabular}{c|c|c|c|c|c}
\hline \multicolumn{2}{c|}{ СТД } & \multicolumn{4}{c}{ RCWA } \\
\hline \multicolumn{2}{c|}{$\theta=30^{\circ}$} & \multicolumn{2}{c|}{$\Psi=30^{\circ}$} & \multicolumn{2}{c}{$\Psi=-30^{\circ}$} \\
\hline$k$ & $\lambda_{k}$ & $k$ & $\lambda_{k}$ & $k$ & $\lambda_{k}$ \\
\hline 78 & 0.398 & 82 & 0.391 & 77 & 0.391 \\
74 & 0.417 & 76 & 0.418 & 71 & 0.418 \\
45 & 0.665 & 48 & 0.645 & 45 & 0.645 \\
41 & 0.727 & 43 & 0.717 & 40 & 0.717 \\
\hline \multicolumn{2}{c|}{$\theta=45^{\circ}$} & \multicolumn{2}{c|}{$\Psi=45^{\circ}$} & \multicolumn{2}{c}{$\Psi=-45^{\circ}$} \\
\hline 87 & 0.3999 & 94 & 0.39 & 84 & 0.393 \\
83 & 0.4172 & 87 & 0.418 & 78 & 0.42 \\
50 & 0.672 & 50 & 0.707 & 45 & 0.705 \\
46 & 0.728 & 49 & 0.721 & 44 & 0.721
\end{tabular}

стве расчетной была принята длина волны $\lambda_{0}=0.55 \mu \mathrm{m}$, а порядок гармоничности $m$ принимался равным 50 . Глубина рельефа $H$ в соответствии с формулой (3) составила $55.7 \mu \mathrm{m}$.

Исследования показали, что хорошая сходимость результатов расчета, а следовательно, и их высокая достоверность достигается при высоте ступеней порядка $(0.3-0.35) \lambda_{\min }$ и числе гармоник ряда Фурье порядка 200. Здесь $\lambda_{\min }-$ минимальная длина волны рабочего спектрального диапазона.

Для того чтобы при разумных затратах машинного времени с достаточной степенью точности сопоставить величины $k$ и $\lambda_{k}$, прогнозируемые формулой (5), c реальными номерами дифракционных порядков и длинами волн, на которые приходятся максимумы и минимумы ДЭ, в начале и в конце спектрального диапазона $(0.39-0.7 \mu \mathrm{m})$ выбирались два поддиапазона 0.39-0.42 $\mu \mathrm{m}$ и 0.64-0.7 $\mu \mathrm{m}$, и расчет ДЭ осуществлялся внутри этих поддиапазонов на длинах волн, отстоящих друг от друга на величину порядка $0.2\left(\lambda_{k}-\lambda_{k+1}\right)$. Сами максимумы и минимумы ДЭ также были зафиксированы, и при этом за минимум принималась суммарная ДЭ двух соседних дифракционных порядков на той длине волны, на которой каждая из суммируемых эффективностей была близка к 0.4.

Для сопоставления углов падения излучения на микроструктуру, приводящих к снижению ДЭ до одного и того же уровня, но полученных двумя различными методами, эти углы обозначены разными буквами: $\theta-$ в рамках СТД и $\Psi$ при использовании RCWA-метода. Это вызвано тем, что в силу асимметрии рельефа модули отрицательного и положительного углов падения излучения (рис. 1), приводящих к снижению ДЭ, оцениваемой RCWA-методом, до одного и того же уровня, могут существенно различаться.

При нормальном падении излучения на элемент $(\theta=\Psi=0)$ соответствующие дифракционные порядки и длины волн, полученные в рамках СТД (табл. 1) и RCWA-методом, совпадают. Однако при наклонном падении излучения и номера дифракционных порядков, и длины волн, полученные различными методами, несколько различаются, что продемонстрировано в табл. 3 на примере микроструктуры с относительным пространственным периодом $\Lambda / H=10$.

Что касается ДЭ, то результаты расчетов, полученные RCWA-методом при $\Psi=0, \pm 30^{\circ}$ и $\pm 45^{\circ}$ для микроструктур с относительными пространственными периодами $\Lambda / H=5$ и $\Lambda / H=10$, сведены в табл. 4 и 5 .

Данные табл. 4 и 5 показывают, что зависимость ДЭ гармонической пилообразной микроструктуры от длины волны, оцениваемая RCWA-методом, имеет ту же самую синусоподобную форму, которую дает и СТД (кривая 4 на рис. 2). При этом с ростом угла падения излучения на элемент, как это и прогнозирует СТД, увеличиваются номера дифракционных порядков, на которые приходятся максимумы ДЭ. Сами максимальное и минимальное значения ДЭ, даваемые как СТД, так и RCWA-методом, не зависят от ширины рабочего спектрального диапазона. В то же время, если прогнозируемые СТД максимальное и минимальное значения ДЭ $\left(\eta_{\max }=1, \eta_{\min } \approx 0.8\right)$ не зависят от угла падения излучения на микроструктуру,

Таблица 4. Максимальные $\eta_{\max }$ и минимальные $\eta_{\min }$ значения ДЭ, полученные RCWA-методом в двух крайних поддиапазонах при $\Lambda / H=5$

\begin{tabular}{c|c|c|c|c}
\hline \multirow{2}{*}{$\begin{array}{l}\text { Угол падения } \\
\text { излучения } \Psi,\end{array}$} & \multicolumn{3}{|c}{ Диапазон длин волн, $\mu \mathrm{m}$} \\
\cline { 2 - 5 } degrees & \multicolumn{2}{|c|}{$0.39-0.42$} & \multicolumn{2}{c}{$0.645-0.725$} \\
\cline { 2 - 5 } & $\eta_{\max }$ & $\eta_{\min }$ & $\eta_{\max }$ & $\eta_{\min }$ \\
\hline 0 & 0.952 & 0.788 & 0.955 & 0.801 \\
+15 & 0.900 & 0.761 & 0.917 & 0.783 \\
-15 & 0.881 & 0.797 & 0.898 & 0.808 \\
+30 & 0.818 & 0.736 & 0.830 & 0.753 \\
-30 & 0.802 & 0.802 & 0.804 & 0.805 \\
+45 & 0.702 & 0.671 & 0.702 & 0.674 \\
-45 & 0.695 & 0.781 & 0.698 & 0.786
\end{tabular}

Таблица 5. Максимальные $\eta_{\max }$ и минимальные $\eta_{\min }$ значения ДЭ, полученные RCWA-методом в двух крайних поддиапазонах при $\Lambda / H=10$

\begin{tabular}{c|c|c|c|c}
\hline \multirow{2}{*}{$\begin{array}{l}\text { Угол падения } \\
\text { излучения }\end{array}$} & \multicolumn{4}{|c}{ Диапазон длин волн, $\mu \mathrm{m}$} \\
\cline { 2 - 5 } degrees & \multicolumn{2}{|c|}{$0.39-0.42$} & \multicolumn{2}{c}{$0.645-0.725$} \\
\cline { 2 - 5 } & $\eta_{\max }$ & $\eta_{\min }$ & $\eta_{\max }$ & $\eta_{\min }$ \\
\hline 0 & 0.957 & 0.7923 & 0.959 & 0.808 \\
+15 & 0.925 & 0.785 & 0.946 & 0.803 \\
-15 & 0.929 & 0.798 & 0.946 & 0.814 \\
+30 & 0.893 & 0.781 & 0.910 & 0.786 \\
-30 & 0.885 & 0.806 & 0.902 & 0.817 \\
+45 & 0.824 & 0.756 & 0.853 & 0.761 \\
-45 & 0.833 & 0.820 & 0.845 & 0.822
\end{tabular}


то значения $\eta_{\max }$ и $\eta_{\min }$, получаемые RCWA-методом, зависят как от модуля, так и от знака угла падения. При этом значения $\eta_{\max }$ уменьшаются с ростом модуля угла падения на величину, зависящую от относительного пространственного периода и знака угла падения. Что касается $\eta_{\min }$, то эта величина в зависимости от модуля и знака угла падения излучения может несколько уменьшаться или, наоборот, увеличиваться. В результате чего наблюдается сглаживание кривой ДЭ или даже ее инвертирование, когда $\eta_{\min }>\eta_{\max }$ (см. последнюю строку табл. 4).

Однако главным является то, что как усредненное по всему рабочему спектральному диапазону, так и наименьшее в пределах этого диапазона значение ДЭ с ростом модуля угла падения излучения на гармоническую киноформную микроструктуру снижаются существенно в меньшей степени, чем в случае двухи трехслойных рельефных дифракционных микроструктур $[9,10]$, имеющих одинаковые с гармонической микроструктурой относительные пространственные периоды.

\section{Хроматизм гармонической киноформной линзы}

Одной из общепринятых характеристик хроматизма первого порядка оптических элементов является коэффициент дисперсии. У обычных рефракционной и киноформной линз этот коэффициент имеет вид

$$
\begin{gathered}
v_{\mathrm{RL}}=\left(n_{\bar{\lambda}}-1\right) /\left(n_{\lambda_{\min }}-n_{\lambda_{\max }}\right), \\
v_{\mathrm{KL}}=\bar{\lambda} /\left(\lambda_{\min }-\lambda_{\max }\right)
\end{gathered}
$$

соответственно [11-13]. В формуле (9) $n_{\bar{\lambda}}, n_{\lambda_{\min }}$ и $n_{\lambda_{\max }}-$ значения показателя преломления материала рефракционной линзы на центральной $\bar{\lambda}$ и крайних длинах волн рабочего спектрального диапазона. Чем больше по модулю коэффициент дисперсии, тем меньше хроматизм. В обобщенном виде, не зависящем от типа оптического элемента, коэффициент дисперсии можно записать как

$$
v=\Phi_{\bar{\lambda}} /\left(\Phi_{\lambda_{\max }}-\Phi_{\lambda_{\min }}\right)
$$

где $\Phi_{\bar{\lambda}}, \Phi_{\lambda_{\max }}$ и $\Phi_{\lambda_{\min }}-$ значения оптической силы элемента (величины, обратной фокусному расстоянию) на соответствующей длине волны.

Если учесть, что в знаменателе формулы (11) должна быть максимальная по модулю разность оптических сил элемента в пределах рабочего спектрального диапазона, то в случае гармонической киноформной линзы, как следует из табл. 1, за максимальную и минимальную длины волн, приводящие к максимальной разности оптических сил, следует принять $\lambda_{\max }=\lambda_{k_{\min }}$ и $\lambda_{\min }=0.5\left(\lambda_{k_{\min }}+\lambda_{k_{\min }+1}\right)$. Тогда центральной длиной волны окажется $\bar{\lambda}=0.5\left(\lambda_{\max }+\lambda_{\min }\right)$. В результате, используя выражение (10), для коэффициента дисперсии гармонической киноформной линзы получим

$$
v_{\mathrm{HKL}}=0.5 \frac{\lambda_{k_{\min +1}}+3 \lambda_{k_{\min }}}{\lambda_{k_{\min +1}}-\lambda_{k_{\min }}} .
$$

Таблица 6. Коэффициенты дисперсии элементов различных типов

\begin{tabular}{l|c|c}
\hline \multirow{2}{*}{$\begin{array}{c}\text { Коэффициент } \\
\text { дисперсии }\end{array}$} & \multicolumn{2}{|c}{ Оптический материал } \\
\cline { 2 - 3 } & РММА & РC \\
\hline$v_{\mathrm{RL}}$ & 25.5 & 12.48 \\
\hline$v_{\mathrm{KL}}$ & \multicolumn{2}{|c}{-1.83} \\
\hline$v_{\mathrm{HKL}}$ & -80.74 & -80.79 \\
$\begin{array}{l}\left(m=50, \lambda_{0}=0.55 \mu \mathrm{m}\right) \\
k_{\min }=38\end{array}$ & &
\end{tabular}

В табл. 6 приведены для сравнения коэффициенты дисперсии элементов вышеперечисленных типов, выполненных из оптических пластмасс РММА и РС, полученные для видимого спектрального диапазона $\left(\lambda_{\min }=0.4 \mu \mathrm{m}\right.$, $\left.\lambda_{\text {max }}=0.7 \mu \mathrm{m}\right)$.

Сразу подчеркнем, что элементы различных типов, включая и гармоническую киноформную линзу, при одинаковых оптических силах $\Phi_{\bar{\lambda}}$ и равных по модулю коэффициентах дисперсии будут иметь одинаковый по модулю продольный хроматизм, т.е. модули максимальных смещений фокальных плоскостей в рабочем спектральном диапазоне будут равными. Следует также обратить внимание на то, что хроматизм гармонической киноформной линзы выбором порядка гармоничности $m$ может управляться в весьма широких пределах и оказаться, например, меньшим, чем у самых легких кронов. Таким образом, в плане хроматизма гармоническая киноформная линза обладает уникальными свойствами, отличающими ее от оптических элементов всех остальных типов.

\section{Заключение}

В настоящей работе в рамках скалярной и строгой теорий дифракции (RCWA-методом) проведено исследование гармонических киноформных линз, пилообразные микроструктуры которых работают в высоких порядках дифракции и имеют глубины рельефа, существенно превышающие расчетную длину волны. Показано, что зависимость ДЭ гармонической пилообразной микроструктуры от длины волны, оцениваемая RCWA-методом, имеет ту же самую синусоподобную форму, которую дает и СТД. При этом с ростом угла падения излучения на элемент, как это и прогнозирует СТД, увеличиваются номера дифракционных порядков, на которые приходятся максимумы ДЭ. Сами максимальное и минимальное значения ДЭ, даваемые как СТД, так и RCWA-методом, не зависят от ширины рабочего спектрального диапазона.

В то же время если прогнозируемые СТД максимальное и минимальное значения ДЭ $\left(\eta_{\max }=1, \eta_{\min } \approx 0.8\right)$ не зависят от угла падения излучения на микроструктуpy, то значения $\eta_{\max }$ и $\eta_{\min }$, получаемые RCWA-методом, 
зависят как от модуля, так и от знака угла падения. Причем как усредненное по всем рабочему спектральному диапазону, так и наименьшее значение ДЭ в пределах этого диапазона с ростом модуля угла падения излучения на гармоническую киноформную микроструктуру снижается существенно в меньшей степени, чем в случае двух- и трехслойных двухрельефных дифракционных микроструктур, имеющих одинаковые с гармонической микроструктурой относительные пространственные периоды. Действительно, если у двух- и трехслойных двухрельефных дифракционных микроструктур модуль допустимого угла падения излучения не превышает $20^{\circ}-25^{\circ}$, то в случае гармонической микроструктуры для этого угла не является предельным и значение в $45^{\circ}$.

Получено выражение для коэффициента дисперсии гармонической киноформной линзы и показано, что выбором порядка гармоничности ее хроматизм может управляться в весьма широких пределах, например, он может оказаться меньшим, чем у самых легких кронов. Таким образом, в плане хроматизма гармоническая киноформная линза обладает уникальными свойствами, которые могут найти применение при решении некоторых нетривиальных оптических задач.

Работа выполнена при финансовой поддержке Российского научного фонда (проект № 17-19-01461).

\section{Список литературы}

[1] Sweeney D.W., Sommargren G.E. // Appl. Opt. 1995. V. 34. N 14. P. 2469

[2] Харитонов С.И., Волотовский С.Г., Хонина С.Н. // Компьютерная оптика. 2016. Т. 40. № 3. С. 331.

[3] Слюсарев Г.Г. // ДАН СССР. 1957. Т. 113. № 4. С. 780.

[4] Radiant Vision Systems. [Электронный ресурс] Режим доступа: www.radiantzemax.com

[5] Buralli D.A., Morris G.M., Rogers J.R. // Appl. Opt. 1989. V. 28. N 5. P. 976.

[6] Greisukh G.I., Danilov V.A., Ezhov E.G., Levin I.A., Stepanov S.A., Usievich B.A. // Opt. Commun. 2015. V. 338. P. 54-57.

[7] Moharam M.G., Gaylord T.K. // JOSA. 1982. V. 72. N 10. P. 1385.

[8] Lyndin N.M. Modal and C Methods Grating Design and Analysis Software. [Электронный ресурс] Режим доступа: http://www.mcgrating.com

[9] Грейсух Г.И., Данилов В.А., Ежов Е.Г., Степанов С.А., Усиевич Б.А. // Оптический журнал. 2015. Т. 82. № 5. С. 56.

[10] Грейсух Г.И., Данилов В.А., Ежсов Е.Г., Степанов С.А., Усиевич Б.А. // Опт. и спектр. 2015. Т. 118. № 6. С. 118.

[11] Чуриловский В.И. Теория хроматизма и аберраций третьего порядка. Л.: Машиностроение, 1968. 311 с.

[12] Грейсух Г.И., Ежсов Е.Г., Степанов С.А. // Компьютерная оптика. 2005. В. 28. С. 60.

[13] Greisukh G.I., Ezhov E.G., Stepanov S.A. // Appl. Opt. 2006. V. 45. N 24. P. 6137. 